\title{
Ceftazidime/Avibactam-Resistant Klebsiella pneumoniae subsp. pneumoniae Isolates in a Tertiary Italian Hospital: Identification of a New Mutation of the Carbapenemase Type 3 (KPC-3) Gene Conferring Ceftazidime/Avibactam Resistance
}

\author{
Carla Fontana ${ }^{1,2} \oplus$, Marco Favaro ${ }^{1}$, Laura Campogiani ${ }^{3}$, Vincenzo Malagnino $\left.{ }^{3} \mathbb{(}\right)$, Silvia Minelli ${ }^{2}$, \\ Maria Cristina Bossa ${ }^{2}$, Anna Altieri ${ }^{2}$, Massimo Andreoni ${ }^{3}$ and Loredana Sarmati ${ }^{3, *(D)}$ \\ 1 Department of Experimental Medicine, “Tor Vergata” University, 00133 Rome, Italy; \\ carla.fontana@uniroma2.it (C.F.); favaro@uniroma2.it (M.F.) \\ 2 Laboratory of Microbiology, Polyclinic of "Tor Vergata", 00133 Rome, Italy; silvia.minelli@ptvonline.it (S.M.); \\ mariacristina.bossa@ptvonline.it (M.C.B.); anna.altieri@ptvonline.it (A.A.) \\ 3 Clinical Infectious Diseases, Department of System Medicine, “Tor Vergata” University, 00133 Rome, Italy; \\ lauracampg@gmail.com (L.C.); malagninovincenzo@gmail.com (V.M.); andreoni@uniroma2.it (M.A.) \\ check for \\ * Correspondence: sarmati@med.uniroma2.it
}

updates

Citation: Fontana, C.; Favaro, M.; Campogiani, L.; Malagnino, V.;

Minelli, S.; Bossa, M.C.; Altieri, A.;

Andreoni, M.; Sarmati, L.

Ceftazidime/Avibactam-Resistant Klebsiella pneumoniae subsp. pneumoniae Isolates in a Tertiary Italian Hospital: Identification of a New Mutation of the Carbapenemase Type 3 (KPC-3) Gene Conferring Ceftazidime/Avibactam Resistance. Microorganisms 2021, 9, 2356. https://doi.org/10.3390/ microorganisms 9112356

Academic Editor: Vijay Pancholi

Received: 31 October 2021

Accepted: 12 November 2021

Published: 15 November 2021

Publisher's Note: MDPI stays neutral with regard to jurisdictional claims in published maps and institutional affiliations.

Copyright: (c) 2021 by the authors. Licensee MDPI, Basel, Switzerland. This article is an open access article distributed under the terms and conditions of the Creative Commons Attribution (CC BY) license (https:// creativecommons.org/licenses/by/ $4.0 /)$.

\begin{abstract}
Several Klebsiella pneumoniae carpabenemase (KPC) gene mutations are associated with ceftazidime/avibactam (CAZ-AVI) resistance. Here, we describe four Klebsiella pneumoniae subsp. pneumoniae CAZ-AVI-resistant clinical isolates, collected at the University Hospital of Tor Vergata, Rome, Italy, from July 2019 to February 2020. These resistant strains were characterized as KPC-3, having the transition from cytosine to thymine (CAC-TAC) at nucleotide position 814, with histidine that replaces tyrosine (H272Y). In addition, two different types of KPC gene mutations were detected. The first one, common to three strains, was the D179Y (G532T), associated with CAZ-AVI resistance. The second mutation, found only in one strain, is a new mutation of the KPC-3 gene: a transversion from thymine to adenine (CTG-CAG) at nucleotide position 553. This mutation causes a KPC variant in which glutamine replaces leucine (Q168L). None of the isolates were detected by a rapid immunochromatographic assay for detection of carbapenemase (NG Biotech, Guipry, France) and were unable to grow on a selective chromogenic medium Carba SMART (bioMerieux, Firenze, Italy). Thus, they escaped common tests used for the prompt detection of Klebsiella pneumoniae KPC-producing.
\end{abstract}

Keywords: ceftazidime/avibactam; multi-drug resistance; antibiotic resistance; beta-lactamase inhibitors resistance; Q168L substitution

\section{Introduction}

Antimicrobial resistance (AMR) is currently one of the major concerns in human health [1,2]. Gram-negative multidrug-resistant (MDR) pathogens represent a major challenge due to the increase in AMR worldwide [3,4] and the high disease burden, with increases in hospital stays, disability-adjusted life years, and mortality [1,2]. The main concern regarding gram-negative AMR includes resistance to carbapenems. A drug pipeline to tackle this threat has been implemented, with two novel $\beta$-lactam betalactamase inhibitor (BLBLI) combinations released in 2015: ceftazidime/avibactam and ceftolozane/tazobactam [3,5-7]. Ceftazidime/avibactam (CAZ-AVI) is a BLBLI combination drug active against carbapenem-resistant Enterobacteriaceae (CRE) [3,6-9]. Avibactam recovers the activity of ceftazidime by inhibiting class $\mathrm{A}$, class $\mathrm{C}$, and some class $\mathrm{D}$ beta-lactamases, hence overcoming resistance due to carbapenemases such as Klebsiella pneumoniae carbapenemase (KPC). However, the combination is not active against class B 
beta-lactamases, such as New-Delhi (NDM) and Verona Integron-encoded (VIM) and other metallo-beta lactamases.

In Italy, the circulation of CRE is endemic [10,11], mainly due to Klebsiella pneumoniae subsp. pneumoniae, but national strategies to address the threat have not yet been fully implemented. CAZ-AVI is frequently used, not only as salvage therapy but also as a firstline choice even in empiric treatments [8]. Since BLBLI introduction, isolation of pathogens with phenotypic resistance to CAZ-AVI has been reported, frequently Klebsiella pneumoniae strains, especially those harboring bla $a_{\mathrm{KPC}}$ gene isolates. In 2015, the first K. pneumoniae subsp. pneumoniae isolate resistant to CAZ-AVI was described in Los Angeles, USA [12], in a patient who was not previously treated with the antibiotic. Subsequently, several other cases of CAZ-AVI resistance have been reported and led to the publication of the 2018 ECDC rapid risk assessment on ceftazidime/avibactam resistance in Enterobacteriaceae [13-19]. The emergence of CAZ-AVI-resistant pathogens is a public health threat, carrying serious consequences for patients, even if surveillance studies report overall low rates of CAZ-AVI resistance in CRE [20]. Data from clinical reports show a strong association with prior CAZAVI exposure and resistant strain selection in more than two-thirds of cases, highlighting a selective pressure caused by the BLBLI itself [20]. Resistance to CAZ-AVI can also emerge from the selection pressure of other antibiotics, such as carbapenems, demonstrating the pivotal role of antimicrobial stewardship programs.

Different mechanisms have been associated with CAZ-AVI resistance, such as mutation of the KPC gene associated with the loss/reduction in KPC enzyme functionality, hyperexpression of the $b l a_{\mathrm{KPC}}$ gene, and finally, the loss of porins [15,21-23]. Some KPC subtypes, such as KPC-3 (nearly 70\% KPC-producing K pneumoniae isolates belonged from a single dominant strain, multilocus sequence type 258 [ST258]; seven variants of the KPC have been reported (KPC 2-8) but most ST258 strains produced KPC-3) and specific $K$. pneumoniae clones, are more likely to develop resistance [13]. A series of mutations in the omega loop of KPC-3 have been documented in patients with microbiological failure to CAZ-AVI treatment, including H272Y, D179Y, V240G, T243M, and EL165-166 [21,24-28]. The D179Y variant, alone or in combination with other mutations, results in restoration of carbapenem in vitro activity of the K. pneumoniae isolates [28,29].

Here we describe the characteristics of four CAZ-AVI-resistant isolates and the identification of a new amino acid substitution in KPC-3 in a K. pneumoniae strain resistant to CAZ-AVI.

\section{Materials and Methods}

\subsection{Bacterial Isolates}

CAZ-AVI-resistant K. pneumoniae subsp. pneumoniae clinical isolates obtained from patients admitted to the University Hospital of Tor Vergata from July 2019 to February 2020 were included in the study. Isolates with resistance mechanisms other than KPC alone were excluded from the study population. The included isolates were named PTV (acronym derived from Policlinic Tor Vergata) and numbered sequentially.

The study was approved by the local ethics committee. Given the retrospective nature of the study, written informed consent was not necessary. The study was conducted in accordance with the principles of the Declaration of Helsinki.

\subsection{Antimicrobial Susceptibility Testing and PCR Analysis}

Antimicrobial susceptibility testing (AST) was performed using ITGN Micronaut panels (Diagnostika Gmbh, Bornheim, Germany, now company of Bruker Daltonics, MA, USA) run on MICRO MIB (Bruker Daltonics, Billerica, MA, USA) and interpreted following the European Committee on Antimicrobial Susceptibility Testing (EUCAST) clinical breakpoint v 9.0. Carba SMART selective chromogenic media (bioMerieux, Firenze, Italy) was used to screen for carbapenemase-producing Enterobacteriaceae (CPE). Identification of carbanemases (KPC, VIM, imipenemase [IMP], NDM, oxacillin-hydrolyzing [OXA] 48) was performed using the immunochromatographic (IC) assay NG CARBA (NG Biotech, 
Guipry, France) according to the manufacturer's instructions. Carbapenemases detected by IC assay were also confirmed by a multiplex real-time PCR probe-based assay (our patent ID MI2014A000327) designed for the simultaneous detection of KPC, OXA-48, VIM, and NDM in a short time (no longer than $90 \mathrm{~min}$ from the extraction of DNA to detection) [30].

\subsection{DNA Extraction from the Isolates}

DNA was extracted starting from a pure culture (after overnight incubation at $37^{\circ} \mathrm{C}$ in an aerobic atmosphere). One colony from a fresh culture of each isolate was suspended in $200 \mu \mathrm{L}$ of G2TM solution (Qiagen Valencia, CA, USA), and then used to extract DNA using the EZ1 Advanced XL Tissue Kit, according to the bacterial protocol recommended by the manufacturers (Qiagen, Valencia, CA, USA). The DNA was eluted in $100 \mu \mathrm{L}$ of elution bufferTM (Qiagen), and $1 \mu \mathrm{L}$ was used to conduct the PCRs as well as sequence analysis.

\subsection{Sequence Analysis}

For the typing of the bla KPC gene, overlapping PCR reactions were performed using the following primer pairs: F-5'CGGAACCATTCGCTAAACTC $3^{\prime}$ and R-3'GGCGGCGTTAT CACTGTATT5'; F-5'CGCCGTGCAATACAGTGATA3' and R-3'CGTTGACGCCCAATCC5 ${ }^{\prime}$ (Goldfarb et al. [31]). Amplification products were purified using the Montage PCR Centrifugal Filter Device (Millipore Corporation, Billerica, MA, USA), and sequencing was performed by Big Dye Terminators V1.1 (Applied Biosystems, Foster City, CA, USA) and migrated with an automated sequencer (ABI Prism 310; Applied Biosystems). Sequences were aligned and compared using the National Center for Biotechnology Information database (http:/ / www.ncbi.nlm.nih.gov, 13 November 2021).

\subsection{Bla KPC Cloning}

The bla KPC gene of KPC-3 with the Q168L substitution (PTV4 isolate) was amplified using the following primers: F prot (ACAGCCGTTACAGCCTCTG) of our design and R874 (Naas et al. [32]). The amplicon, purified using the QIAquick PCR Purification Kit (Qiagen Valencia, CA, USA), was inserted into the pGem Teasy Vector System II (Promega; Milan, Italy) following the manufacturer's instruction.

The chimeric plasmid was used to transform Escherichia coli HB101 competent cells, included in the kit (Promega). Transformants were selected on SuperCAZ/AVI medium (Lifilchem, Roseto degli Abbruzzi, Italy) and screened by multiplex real-time PCR to confirm the presence of blaKPC genes. Finally, to establish the CAZAVI-MIC, transformants were tested using a microbroth dilution test (ITGN, Biomedical service) as above reported.

\section{Results}

Seven CAZ-AVI-resistant (CAZ-AVI-R) isolates were identified in biological samples collected from patients hospitalized from 3 July 2019 to 5 February 2020 at Policlinic Tor Vergata University Hospital, Rome, Italy. Isolates were all Klebsiella pneumoniae, of which two expressed class B-beta-lactamases (non-KPC) and one had more than one resistance mechanism. Four isolates (57.1\%) expressed only the KPC genotype, representing our final study population (Figure 1). 


\begin{tabular}{|c|}
\hline Klebsiella pneumoniae subsp. pneumoniae \\
with Ceftazidime/Avibactam resistance \\
(3 July 2019 to 5 February 2020) \\
7 isolates \\
\hline
\end{tabular}
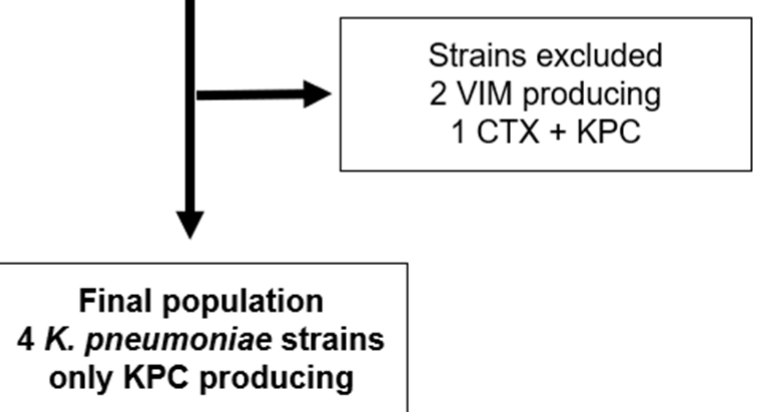

Figure 1. Flow chart showing K. pneumoniae CAZ-AVI-R strains selection criteria.

All patients had CAZ-AVI-sensitive K. pneumoniae (CAZ-AVI-S) prior to isolating a CAZ-AVI-R strain. Sample details and timing of collection are shown in Table 1. All but one CAZ-AVI-R isolates were resistant to carbapenems exhibiting an MIC for meropenem ranging from 16 to $>64 \mathrm{mg} / \mathrm{L}$. The time elapsed from the isolation of a CAZ-AVI-R in patients with previous isolates of CAZ-AVI-S ranged from 1 to 52 days. The antimicrobial susceptibility results for the four CAZ-AVI-R isolates are shown in Table 2.

Table 1. Details on CAZ-AVI-R and CAZ-AVI-S Klebsiella pneumoniae strains.

\begin{tabular}{|c|c|c|c|c|c|c|}
\hline $\begin{array}{l}\text { Isolate } \\
\text { ID }\end{array}$ & $\begin{array}{l}\text { Admission } \\
\text { Ward }\end{array}$ & Type of Specimen & $\begin{array}{l}\text { Date of } \\
\text { Specimen } \\
\text { Collection }\end{array}$ & $\begin{array}{l}\text { Meropenem } \\
\text { MIC }\end{array}$ & $\begin{array}{l}\text { CAZ-AVI } \\
\text { MIC }\end{array}$ & $\begin{array}{c}\text { Time from Isolation of } \\
\text { CAZ-AVI-R in Patients with } \\
\text { Previous CAZ-AVI-S }\end{array}$ \\
\hline \multirow{2}{*}{ PTV1 } & \multirow{2}{*}{$\begin{array}{l}\text { Vascular } \\
\text { surgery }\end{array}$} & Urine culture & $30 / 04 / 2019$ & $>16$ & 2 & \multirow{2}{*}{52 days } \\
\hline & & Rectal swab & $21 / 06 / 2019$ & 4 & $>8$ & \\
\hline \multirow[b]{2}{*}{ PTV2 } & \multirow{2}{*}{$\begin{array}{l}\text { Cardio } \\
\text { surgical } \\
\text { intensive } \\
\text { care }\end{array}$} & $\begin{array}{l}\text { Wound swab } \\
\text { (sternotomy) }\end{array}$ & $22 / 07 / 2019$ & $>16$ & $\leq 1$ & \multirow{2}{*}{44 days } \\
\hline & & $\begin{array}{c}\text { Bronchoalveolar } \\
\text { lavage }\end{array}$ & 04/09/2019 & 2 & $>8$ & \\
\hline \multirow[b]{2}{*}{ PTV3 } & \multirow{2}{*}{$\begin{array}{l}\text { Transplant } \\
\text { surgery }\end{array}$} & Blood culture & $15 / 11 / 2019$ & $\leq 0.125$ & 8 & \multirow[b]{2}{*}{1 day } \\
\hline & & $\begin{array}{l}\text { Intrabdominal } \\
\text { abscess }\end{array}$ & $16 / 11 / 2019$ & 2 & 64 & \\
\hline \multirow{2}{*}{ PTV4 } & \multirow{2}{*}{$\begin{array}{l}\text { Medicine } \\
\text { ward }\end{array}$} & Urine culture & $01 / 02 / 2020$ & $>64$ & 2 & \multirow{2}{*}{4 days } \\
\hline & & Blood culture & $05 / 02 / 2020$ & $\leq 0.125$ & 32 & \\
\hline
\end{tabular}

Table 2. Antimicrobial susceptibilities of the four CAZ-AVI-R strains.

\begin{tabular}{ccccccccccccccc}
\hline Isolate ID & \multicolumn{10}{c}{ MICs mg/L } & \multicolumn{10}{c}{ MIP } & CEF & CTZ & CAA & CFT & CIP & COL & GN & IMI & MEM & LEV & PZT & TSU \\
\hline & AK & $>8$ & $>32$ & $>8$ & $>8$ & $>8$ & $\leq 1$ & 0.5 & 4 & 4 & $>8$ & $>128$ & $>8$ \\
\hline PTV2 & $\leq 4$ & $>8$ & $>32$ & $>8$ & $>8$ & $>8$ & $\leq 1$ & $\leq 0.25$ & $\leq 1$ & 2 & $>8$ & $>128$ & $>8$ \\
\hline PTV3 & $\leq 4$ & $>16$ & $>64$ & 64 & $>64$ & $>1$ & 1 & $>8$ & NA & 2 & $>8$ & 128 & $>8$ \\
\hline PTV4 & $\leq 4$ & $>16$ & $>64$ & 32 & 64 & $>1$ & $<0.5$ & $>8$ & NA & $\leq 0.125$ & 4 & 16 & $>8$ \\
\hline
\end{tabular}

AK: Amikacin; CEF: Cefepime; CTZ: Ceftazidime; CAA: Ceftazidime/Avibactam; CFT: Ceftolozane/Tazobactam; CIP: Ciprofloxacin; COL: Colistin; GN: Gentamycin; IMI: Imipenem; MEM: Meropenem; LEV: Levofloxacin; PZT: Piperacillin/Tazobactam; TSU: Trimethoprim/Sulfamethoxazole; MIC: minimum inhibitory concentration. NA: not available 
All CAZ-AVI-R strains were unable to grow on the selective chromogenic medium Carba SMART (bioMerieux, Firenze, Italy). All CAZ-AVI-R isolates were investigated by immunochromatographic assay (NG CARBA Biotech, Guipry, France), and the KPC enzyme was undetectable. In contrast, molecular assays detected the presence of the bla $\mathrm{KPC}_{\mathrm{KP}}$ gene in all strains, and the KPC-3 variants were identified by sequencing (Table 3).

Table 3. Details on CAZ-AVI-R strains.

\begin{tabular}{|c|c|c|c|c|}
\hline Isolate ID & Type of Specimen & $\begin{array}{c}\text { Carbapenemases } \\
\text { Detected }\end{array}$ & Mutation & Amino Acid Substitution \\
\hline PTV1 & Rectal swab & bla $\mathrm{KPC}-\mathrm{KPC} 3$ & $\begin{array}{l}\text { C814T } \\
\text { G523T }\end{array}$ & $\begin{array}{l}\text { H272Y-Histidine vs. Tyrosine } \\
\text { D179Y-Aspartic Ac vs. Tyrosine }\end{array}$ \\
\hline PTV2 & Bronchoalveolar lavage & bla $\mathrm{KPC}^{-\mathrm{KPC} 3}$ & $\begin{array}{l}\text { C814T } \\
\text { G523T }\end{array}$ & $\begin{array}{l}\text { H272Y-Histidine vs. Tyrosine } \\
\text { D179Y-Aspartic Ac vs. Tyrosine }\end{array}$ \\
\hline PTV3 & $\begin{array}{l}\text { Intrabdominal abscess } \\
\text { drainage }\end{array}$ & bla KPC-KPC3 & $\begin{array}{l}\text { C814T } \\
\text { G523T }\end{array}$ & $\begin{array}{l}\text { H272Y-Histidine vs. Tyrosine } \\
\text { D179Y-Aspartic Ac vs. Tyrosine }\end{array}$ \\
\hline PTV4 & Blood culture & bla KPC-KPC3 & $\begin{array}{c}\text { C814T } \\
\text { 553/CTG-CAG }\end{array}$ & $\begin{array}{l}\text { H272Y-Histidine vs. Tyrosine } \\
\text { Q168L_Glutamine vs. Leucine }\end{array}$ \\
\hline
\end{tabular}

For all isolates, the bla KPC gene showed a mutation at position 814 that was a transition from cytosine to thymine CAC-TAC (UAC), identifying the KPC-3 variant, in which histidine replaced tyrosine (H272Y). PTV1-3 exhibited a substitution D179Y, owing to a single base mutation G532T (33). Sequence analysis of the KPC gene in the PTV4 strain showed an additional mutation at position 553, a transversion from thymine to adenine (CTG-CAG), that caused a variant in which glutamine replaced leucine (Q168L) (Table 3). The complete bla $\mathrm{KPC}_{\mathrm{C}}$ gene sequencing was deposited into the NCBI (accession number MT939316).

The bla $\mathrm{KPC}_{\mathrm{C}}$ cloning studies demonstrated that transformants carrying the Q168L mutation were resistant to CAZAVI, being the CAZAVI-MIC equal to $32 \mu \mathrm{g} / \mathrm{mL}$ in a microbroth dilution test (data not shown).

Figure 2 shows the 3D structure of mutant and native KPC enzymes of PTV4 strain.
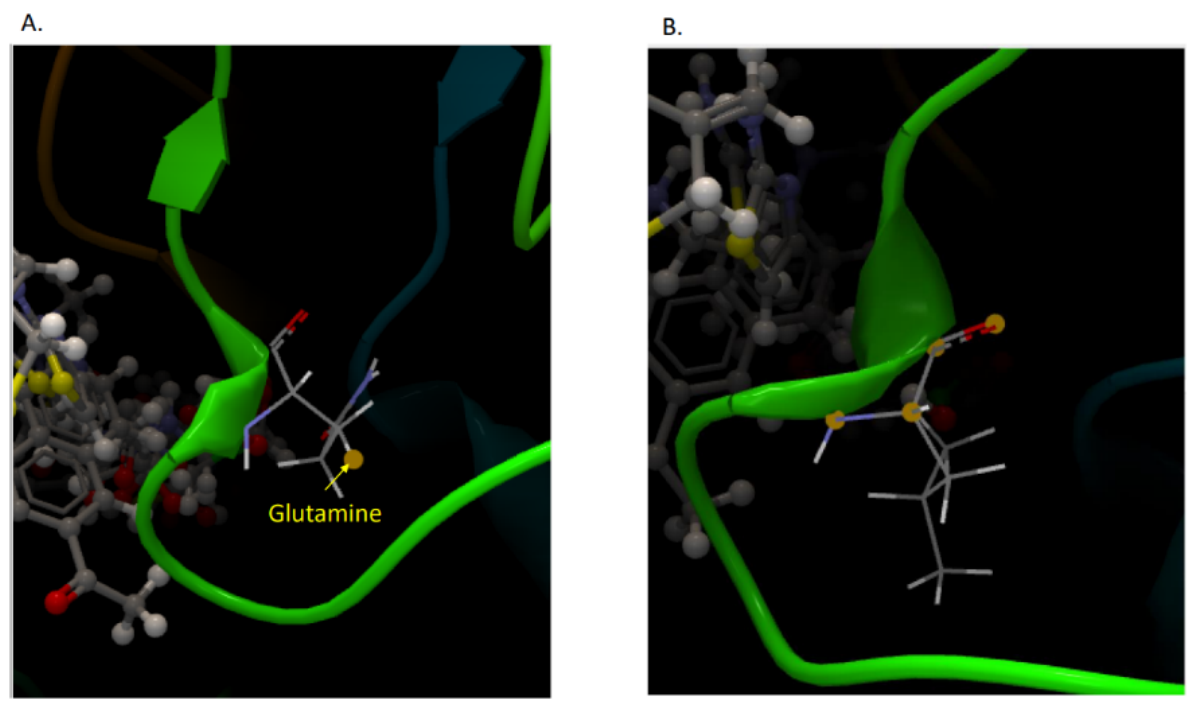

Figure 2. Three-dimensional (3D) images of KPC-3 of PTV4 strain. (A) KPC-3 with the mutation in which glutamine replaces leucine (Q168L). (B) Wild type. The 3D images have been obtained using a free available bioinformatics web server at https:/ / www.reading.ac.uk/bioinf/servlets/nFOLD/IntFOLD6 results.jsp?time=16_5_30_665_7-10-2020_All\&md5=295b5b0r3v0mlj1k\&targetname=striatin-4\#TS (accessed on 13 November 2021). 


\section{Discussion}

We reported data on four genotypically characterized Klebsiella pneumoniae KPCproducing strains resistant to CAZ-AVI, one of which showed a new mutation conferring resistance to CAZ-AVI.

The emergence of K. pneumoniae CAZ-AVI-R is a worrisome issue, posing important therapeutic and public health challenges [15,19-22]. Risk factors for resistance selection have not yet been fully elucidated, however, several authors have identified suboptimal antibiotic exposure as one of the main factors associated with CAZ-AVI-R emergence [13]. In this study, K. pneumoniae strains were obtained from patients with a previous long exposure time to CAZ-AVI. However, the development of isolates resistant to CAZ-AVI occurred after 4 days of therapy in one case, therefore, it is difficult to establish the critical duration of exposure to CAZ-AVI associated with resistance. Strains of K. pneumoniae resistant to CAZ-AVI have been isolated from anatomical sites other than those of susceptible strains; therefore, it is reasonable to hypothesize an easier selection of resistant CAZ-AVI isolates in anatomical sites less accessible to the antibiotic, where the presence of subtherapeutic drug concentrations is conceivable. However, the limited number of strains studied, and the lack of pharmacokinetic data do not allow definitive conclusions on this issue.

As reported by other studies [20,33-37], restoration of susceptibility to meropenem was noticed in K. pneumoniae isolates switching to CAZ-AVI resistance. This phenomenon has been related to amino acid substitutions and conformational changes in the active site of carbapenemase enzymes, that resulted in the restoration of low minimum inhibitory concentrations (MICs) to meropenem. The use of meropenem in these cases was, however, not recommended, as it is often followed by the rapid restoration of resistance to the drug $[20,21,37,38]$.

Our study specifically addresses CAZ-AVI resistance in Klebsiella pneumoniae KPC, highlighting the presence of important diagnostic challenges. None of the CAZ-AVI-R strains were detected as KPC-producing strains by the immunochromatographic assay, and they did not grow on a selective chromogenic medium. This behavior, already described by Antonelli and colleagues [29], raises the serious problem of a shortage of diagnostic tools that clinical microbiology laboratories should be aware of. Limitations of the immunochromatographic assay should be considered when testing Klebsiella pneumoniae isolates, especially in patients receiving treatment with CAZ-AVI. The rapidity of immunochromatographic tests has important clinical value; hence, the validity of the test should be thoroughly assessed. Accurate epidemiological studies and the identification of risk factors for the development of resistance to CAZ-AVI could help to identify target populations in which use specific molecular assays instead of relying on rapid diagnostic tools. In situations where there is a strong suspicion of possible resistance to CAZ-AVI, genotypic testing in combination with the resistance profile remains the only reliable option for detecting the resistance mechanism, to be confirmed by sequencing the allelic variant.

Finally, in addition to the mutation at position 532, a transition that causes aspartic acid to replace tyrosine (D179Y) known to be associated with CAZ-AVI resistance in KPC, the PTV4 isolate presented another site mutation at position 553. This mutation, never described before, is a transversion from thymine to adenine (CTG-CAG). The mutation causes a KPC variant in which glutamine replaces leucine (Q168L). Observing 3D images of the KPC variant, we can speculate that the amino acid substitution causes a change in the omega loop of KPC-3, which causes resistance to CAZ-AVI. The bla KPC $_{\text {cloning }}$ demonstrated that the new mutation was able to confer resistance to CAZ-AVI being the MIC of CAZ-AVI, performed on transformants in a microbroth dilution test, equal to $32 \mu \mathrm{g} / \mathrm{mL}$.

Continuous reporting of new mutations in the KPC gene is essential, and the identification of risk factors for CAZ-AVI resistance is useful in optimizing the use of the new BLBLI for the treatment of K. pneumoniae KPC-producing infections. Microbiologists should be particularly vigilant in monitoring resistance in patients infected and colonized with $K$. pneumoniae KPC-producing strains by using all available diagnostic tools. National studies 
with a greater number of patients would allow us to better identify the risk factors for CAZ-AVI resistance and address the challenge of emerging MDR pathogens.

Author Contributions: Conceptualization, C.F., M.F., L.C. and L.S.; methodology, C.F., M.F. and L.C.; data curation, C.F., M.F., L.C., S.M., M.C.B. and A.A.; writing-original draft preparation, C.F., L.C. and L.S.; writing-review and editing, L.S., V.M. and M.A.; visualization, C.F. and M.F. All authors have read and agreed to the published version of the manuscript.

Funding: This research received no external funding.

Institutional Review Board Statement: The study was conducted according to the guidelines of the Declaration of Helsinki and approved by the Policlinico Tor Vergata Ethics Committee (experimentation register 177.21, 22 September 2021).

Informed Consent Statement: Due to the retrospective nature of the study no specific ethics committee's consent was required [Determination of the Italian Drug Agency (AIFA) of 20 March 2008]. With respect to privacy, personal information was treated in a confidential manner, and clinical data were anonymized.

Data Availability Statement: Analyzed data are available in datasets generated during the study.

Conflicts of Interest: M.A. and L.S. received travel grants from Gilead, Merck, Gilead, Bristol, and Pfizer, payment for lectures from Merck, Bristol, and Abbvie, consulting fees from Angelini SpA. and research funding on HIV and Hepatitis from Gilead. All the authors declare no conflict of interest. The funders had no role in the design of the study; in the collection, analyses, or interpretation of data; in the writing of the manuscript, or in the decision to publish the results.

\section{References}

1. Tacconelli, E.; Pezzani, M.D. Public health burden of antimicrobial resistance in Europe. Lancet Infect. Dis. 2019, 19, 4-6. [CrossRef]

2. Prestinaci, F.; Pezzotti, P.; Pantosti, A. Antimicrobial resistance: A global multifaceted phenomenon. Pathog. Glob. Health 2015, 109, 309-318. [CrossRef]

3. Koulenti, D.; Song, A.; Ellingboe, A.; Abdul-Aziz, M.H.; Harris, P.; Gavey, E.; Lipman, J. Infections by multidrug-resistant Gram-negative Bacteria: What's new in our arsenal and what's in the pipeline? Int. J. Antimicrob. Agents 2019, 53, 211-224. [CrossRef] [PubMed]

4. WHO I Global Priority List of Antibiotic-Resistant Bacteria to Guide Research, Discovery, and Development of New Antibiotics. Available online: http://www.who.int/medicines/publications/global-priority-list-antibiotic-resistant-bacteria/en/ (accessed on 22 February 2021).

5. Rodríguez-Baño, J.; Gutiérrez-Gutiérrez, B.; Machuca, I.; Pascual, A. Treatment of Infections Caused by Extended-Spectrum-BetaLactamase-, AmpC-, and Carbapenemase-Producing Enterobacteriaceae. Clin. Microbiol. Rev. 2018, 31, e00079-17. [CrossRef] [PubMed]

6. Bonomo, R.A.; Burd, E.M.; Conly, J.; Limbago, B.M.; Poirel, L.; Segre, J.A.; Westblade, L.F. Carbapenemase-Producing Organisms: A Global Scourge. Clin. Infect. Dis. 2018, 66, 1290-1297. [CrossRef] [PubMed]

7. Torres, A.; Zhong, N.; Pachl, J.; Timsit, J.-F.; Kollef, M.; Chen, Z.; Song, J.; Taylor, D.; Laud, P.J.; Stone, G.G.; et al. Ceftazidimeavibactam versus meropenem in nosocomial pneumonia, including ventilator-associated pneumonia (Reprove): A randomised, double-blind, phase 3 non-inferiority trial. Lancet Infect. Dis. 2018, 18, 285-295. [CrossRef]

8. Temkin, E.; Torre-Cisneros, J.; Beovic, B.; Benito, N.; Giannella, M.; Gilarranz, R.; Jeremiah, C.; Loeches, B.; Machuca, I.; JiménezMartín, M.J.; et al. Ceftazidime-Avibactam as Salvage Therapy for Infections Caused by Carbapenem-Resistant Organisms. Antimicrob. Agents Chemother. 2017, 61, e01964-16. [CrossRef]

9. Tumbarello, M.; Trecarichi, E.M.; Corona, A.; DERosa, F.G.; Bassetti, M.; Mussini, C.; Menichetti, F.; Viscoli, C.; Campoli, C.; Venditti, M.; et al. Efficacy of Ceftazidime-Avibactam Salvage Therapy in Patients with Infections Caused byKlebsiella pneumoniaeCarbapenemase-producingK. pneumoniae. Clin. Infect. Dis. 2019, 68, 355-364. [CrossRef]

10. Piano Nazionale di Contrasto dell'Antimicrobico-Resistenza (PNCAR) 2017-2020. Available online: https://www.salute.gov.it/ portale/documentazione/p6_2_2_1.jsp?id=2660 (accessed on 18 June 2021).

11. Antimicrobial Resistance in the EU/EEA (EARS-Net)_Annual Epidemiological Report 2019. Available online: https:/ / drive. google.com/file/d/1LEyrSMcGv2NofDshqatH8WeIUIzy2ZiF/view (accessed on 22 June 2021).

12. Humphries, R.M.; Yang, S.; Hemarajata, P.; Ward, K.W.; Hindler, J.A.; Miller, S.A.; Gregson, A. First Report of CeftazidimeAvibactam Resistance in a KPC-3-Expressing Klebsiella pneumoniae Isolate. Antimicrob. Agents Chemother. 2015, 59, 6605-6607. [CrossRef]

13. Rapid Risk Assessment: Emergence of Resistance to Ceftazidime-Avibactam in Carbapenem-Resistant Enterobacteriaceae. 13 June 2018. Available online: https:/ / www.ecdc.europa.eu/en/publications-data/rapid-risk-assessment-emergence-resistanceceftazidime-avibactam-carbapenem (accessed on 26 June 2021). 
14. Shields, R.K.; Potoski, B.A.; Haidar, G.; Hao, B.; Doi, Y.; Chen, L.; Press, E.G.; Kreiswirth, B.N.; Clancy, C.J.; Nguyen, M.H. Clinical Outcomes, Drug Toxicity, and Emergence of Ceftazidime-Avibactam Resistance Among Patients Treated for CarbapenemResistant Enterobacteriaceae Infections: Table 1. Clin. Infect. Dis. 2016, 63, 1615-1618. [CrossRef]

15. Gaibani, P.; Campoli, C.; Lewis, R.E.; Volpe, S.L.; Scaltriti, E.; Giannella, M.; Pongolini, S.; Berlingeri, A.; Cristini, F.; Bartoletti, M.; et al. In vivo evolution of resistant subpopulations of KPC-producing Klebsiella pneumoniae during ceftazidime/avibactam treatment. J. Antimicrob. Chemother. 2018, 73, 1525-1529. [CrossRef] [PubMed]

16. Munoz-Price, L.S.; Reeme, A.E.; Buchan, B.W.; Mettus, R.T.; Mustapha, M.M.; Van Tyne, D.; Shields, R.K.; Doi, Y. Patient-to-Patient Transmission of Klebsiella pneumoniae Carbapenemase Variants with Reduced Ceftazidime-Avibactam Susceptibility. Antimicrob. Agents Chemother. 2019, 63, e00955-19. [CrossRef] [PubMed]

17. Giddins, M.J.; Macesic, N.; Annavajhala, M.K.; Stump, S.; Khan, S.; McConville, T.H.; Mehta, M.; Gomez-Simmonds, A.; Uhlemann, A.C. Successive Emergence of Ceftazidime-Avibactam Resistance through Distinct Genomic Adaptations in blaKPC2-Harboring Klebsiella pneumoniae Sequence Type 307 Isolates. Antimicr Agents Chemother. 2018, 62, e02101-17. [CrossRef]

18. Galani, I.; Antoniadou, A.; Karaiskos, I.; Kontopoulou, K.; Giamarellou, H.; Souli, M. Genomic characterization of a KPC-23producing Klebsiella pneumoniae ST258 clinical isolate resistant to ceftazidime-avibactam. Clin. Microbiol. Infect. 2019, 25, 763.e5-763.e8. [CrossRef] [PubMed]

19. Göttig, S.; Frank, D.; Mungo, E.; Nolte, A.; Hogardt, M.; Besier, S.; Wichelhaus, T.A. Emergence of ceftazidime/avibactam resistance in KPC-3-producing Klebsiella pneumoniae in vivo. J. Antimicrob. Chemother. 2019, 74, 3211-3216. [CrossRef] [PubMed]

20. Di Bella, S.; Giacobbe, D.R.; Maraolo, A.E.; Viaggi, V.; Luzzati, R.; Bassetti, M.; Luzzaro, F.; Principe, L. Resistance to ceftazidime/avibactam in infections and colonisations by KPC-producing Enterobacterales: A systematic review of observational clinical studies. J. Glob. Antimicrob. Resist. 2021, 25, 268-281. [CrossRef] [PubMed]

21. Shields, R.K.; Chen, L.; Cheng, S.; Chavda, K.D.; Press, E.G.; Snyder, A.; Pandey, R.; Doi, Y.; Kreiswirth, B.N.; Nguyen, M.H.; et al. Emergence of Ceftazidime-Avibactam Resistance Due to Plasmid-Borne blaKPC-3 Mutations during Treatment of Carbapenem-Resistant Klebsiella pneumoniae Infections. Antimicrob. Agents Chemother. 2017, 61, e02097-16. [PubMed]

22. Nelson, K.; Hemarajata, P.; Sun, D.; Rubio-Aparicio, D.; Tsivkovski, R.; Yang, S.; Sebra, R.; Kasarskis, A.; Nguyen, H.; Hanson, B.M.; et al. Resistance to Ceftazidime-Avibactam Is Due to Transposition of KPC in a Porin-Deficient Strain of Klebsiella pneumoniae with Increased Efflux Activity. Antimicrob. Agents Chemother. 2017, 61, e00989-17. [CrossRef] [PubMed]

23. Antinori, E.; Unali, I.; Bertoncelli, A.; Mazzariol, A. Klebsiella pneumoniae carbapenemase (KPC) producer resistant to ceftazidimeavibactam due to a deletion in the blaKPC3 gene. Clin. Microbiol. Infect. 2020, 26, 946.e1-946.e3. [CrossRef]

24. Livermore, D.M.; Warner, M.; Jamrozy, D.; Mushtaq, S.; Nichols, W.W.; Mustafa, N.; Woodford, N. In Vitro Selection of Ceftazidime-Avibactam Resistance in Enterobacteriaceae with KPC-3 Carbapenemase. Antimicrob. Agents Chemother. 2015, 59, 5324-5330. [CrossRef]

25. Haidar, G.; Clancy, C.J.; Shields, R.K.; Hao, B.; Cheng, S.; Nguyen, M.H. Mutations in blaKPC-3 That Confer CeftazidimeAvibactam Resistance Encode Novel KPC-3 Variants That Function as Extended-Spectrum $\beta$-Lactamases. Antimicrob. Agents Chemother. 2017, 61, e02534-16. [CrossRef] [PubMed]

26. Castanheira, M.; Arends, S.J.R.; Davis, A.P.; Woosley, L.N.; Bhalodi, A.A.; MacVane, S.H. Analyses of a Ceftazidime-AvibactamResistant Citrobacter freundii Isolate Carrying blaKPC-2 Reveals a Heterogenous Population and Reversible Genotype. $m S p h e r e$ 2018, 3, e00408-18. [CrossRef] [PubMed]

27. Hemarajata, P.; Humphries, R.M. Ceftazidime/avibactam resistance associated with L169P mutation in the omega loop of KPC-2. J. Antimicrob. Chemother. 2019, 74, 1241-1243. [CrossRef]

28. Venditti, C.; Nisii, C.; Ballardini, M.; Meledandri, M.; Di Caro, A. Identification of L169P mutation in the omega loop of KPC-3 after a short course of ceftazidime/avibactam. J. Antimicrob. Chemother. 2019, 74, 2466-2467. [CrossRef]

29. Antonelli, A.; Giani, T.; Di Pilato, V.; Riccobono, E.; Perriello, G.; Mencacci, A.; Rossolini, G.M. KPC-31 expressed in a ceftazidime/avibactam-resistant Klebsiella pneumoniae is associated with relevant detection issues. J. Antimicrob. Chemother. 2019, 74, 2464-2466. [CrossRef] [PubMed]

30. Favaro, M.; Sarti, M.; Fontana, C. Multiplex real-time PCR probe-based for identification of strains producing: OXA48, VIM, KPC and NDM. World J. Microbiol. Biotechnol. 2014, 30, 2995-3001. [CrossRef] [PubMed]

31. Goldfarb, D.; Harvey, S.; Jessamine, K.; Jessamine, P.; Toye, B.; Desjardins, M. P70 Detection of plasmid mediated KPC-producing Klebsiella pneumo-niae in Ottawa, Canada: Evidence of intra-hospital transmission. Int. J. Antimicrob. Agents 2009, 34, S49. [CrossRef]

32. Naas, T.; Cuzon, G.; Villegas, M.V.; Lartigue, M.F.; Quinn, J.P.; Nordmann, P. Genetic structures at the origin of acquisition of the beta-lactamase blaKPC gene. Antimicrob. Agents Chemother. 2008, 52, 1257-1263. [CrossRef]

33. Shields, R.K.; Nguyen, M.H.; Press, E.G.; Chen, L.; Kreiswirth, B.N.; Clancy, C.J. Emergence of ceftazidime-avibactam resistance and restoration of carbapenem susceptibility in Klebsiella pneumoniae carbapenemase-producing K. pneumoniae: A case report and review of literature. Open Forum. Infect. Dis. 2017, 4, ofx101. [CrossRef]

34. Li, D.; Li, K.; Dong, H.; Ren, D.; Gong, D.; Jiang, F.; Shi, C.; Li, J.; Zhang, Q.; Yan, W.; et al. Ceftazidime-Avibactam Resistance in Klebsiella pneumoniae Sequence Type 11 Due to a Mutation in Plasmid-Borne blakpc-2 to blakpc-33, in Henan, China. Infect. Drug Resist. 2021, 14, 1725-1731. [CrossRef] 
35. Winkler, M.L.; Papp-Wallace, K.M.; Bonomo, R.A. Activity of ceftazidime/avibactam against isogenic strains of Escherichia coli containing KPC and SHV $\beta$-lactamases with single amino acid substitutions in the omega-loop. J. Antimicrob. Chemother. 2015, 70, 2279-2286. [CrossRef] [PubMed]

36. Compain, F.; Arthur, M. Impaired Inhibition by Avibactam and Resistance to the Ceftazidime-Avibactam Combination Due to the D 179 Y Substitution in the KPC-2 $\beta$-Lactamase. Antimicrob. Agents Chemother. 2017, 61, e00451-17. [CrossRef]

37. Zhang, P.; Shi, Q.; Hu, H.; Hong, B.; Wu, X.; Du, X.; Akova, M.; Yu, Y. Emergence of ceftazidime/avibactam resistance in carbapenem-resistant Klebsiella pneumoniae in China. Clin. Microbiol. Infect. 2020, 26, 124.e1-124.e4. [CrossRef] [PubMed]

38. Woodford, N.; Tierno, P.M., Jr.; Young, K.; Tysall, L.; Palepou, M.F.; Ward, E.; Painter, R.E.; Suber, D.F.; Shungu, D.; Silver, L.L.; et al. Outbreak of Klebsiella pneumoniae producing a new carbapenem-hydrolyzing class A beta-lactamase, KPC-3, in a New York Medical Center. Antimicrob. Agents Chemother. 2004, 48, 4793-4799. [CrossRef] [PubMed] 